\title{
Investigation on Potential-Induced Degradation in a 50 MWp Crystalline Silicon Photovoltaic Power Plant
}

\author{
Jingsheng Huang, ${ }^{1,2}$ Hongtao $\mathrm{Li}^{2}$ Yaojie Sun $\mathbb{D},{ }^{1}$ He Wang $\mathbb{D},{ }^{3}$ and Hong Yang $\mathbb{D}^{3}$ \\ ${ }^{1}$ School of Information Science and Technology, Fudan University, Shanghai 200433, China \\ ${ }^{2}$ State Key Laboratory of Operation and Control of Renewable Energy \& Storage Systems, China Electric Power Research Institute, \\ Nanjing 210003, China \\ ${ }^{3}$ MOE Key Laboratory for Nonequilibrium Synthesis and Modulation of Condensed Matter, School of Science, \\ Xi'an Jiaotong University, Xi'an 710049, China
}

Correspondence should be addressed to Yaojie Sun; yjsun@fudan.edu.cn and Hong Yang; hongy126@126.com

Received 8 June 2018; Revised 26 July 2018; Accepted 14 August 2018; Published 28 October 2018

Academic Editor: Chun-Sheng Jiang

Copyright (c) 2018 Jingsheng Huang et al. This is an open access article distributed under the Creative Commons Attribution License, which permits unrestricted use, distribution, and reproduction in any medium, provided the original work is properly cited.

\begin{abstract}
The regular performance deterioration of P-type crystalline silicon solar modules and module strings caused by potential-induced degradation in a photovoltaic power plant was found in the field. The PID-affected solar modules dismounted from the photovoltaic power plant were further investigated systematically in the laboratory. For the first time, we found that the neutral point of voltage in a module string moved forward to the positive pole for a PID-affected module string as time goes on. Even if low positive voltage is applied to a PID-prone module, it could cause PID. The thermographic and electroluminescence (EL) images of a PID-affected module string also exhibit a regular degradation pattern. This is in good agreement with the measured power loss of the dismounted solar modules under standard test conditions. The results obtained in this paper show that the maximum power degradation rate of solar modules was as high as $53.26 \%$ after only one year of operation because of PID in the field. Due to the vast amount of solar modules and incomplete recovery, this is a terrible catastrophe for the owner of a power plant and module producer.
\end{abstract}

\section{Introduction}

The crystalline silicon solar module has been considered to possess high reliability over 25 years with about a $0.8 \%$ power degradation rate per year $[1,2]$. However, in recent years, a catastrophic power degradation caused by potential-induced degradation (PID) has attracted considerable attention from power plant owners, module producers, and researchers. Some investigators thought that several module components, such as the front cover substrate, encapsulate, silicon nitride (SiNx:H) film, rain, and soiling are related with PID, and they suggested preventing PID on the level of solar cells and module manufacturing [3-6]. But other authors think that the metal ions from the soda lime front cover glass are a key reason for PID. These sodium decorated stacking faults are identified as the primary cause of potential-induced degradation [7-10].
Up to now, there always exist some debates about the mechanism of PID, and there are also no effective measures to prevent PID. Yang et al. even reported the performance deterioration of substrings for PID-affected modules [11]. In this paper, the regular performance deterioration of Ptype crystalline silicon solar modules and module strings caused by potential-induced degradation in a photovoltaic power plant was found in the field. The PID-affected solar modules dismounted from the photovoltaic power plant were further investigated systematically in the laboratory. A detailed analysis on the PID that occurred in the $50 \mathrm{MWp}$ crystalline silicon solar power plant is presented in this contribution. We find that the neutral point of voltage in a module string moved forward to the positive pole for a PID-affected module string as time goes on for the first time. Even if low positive voltage is applied to the PID-prone module, it could cause PID. The regular degradation patterns of 


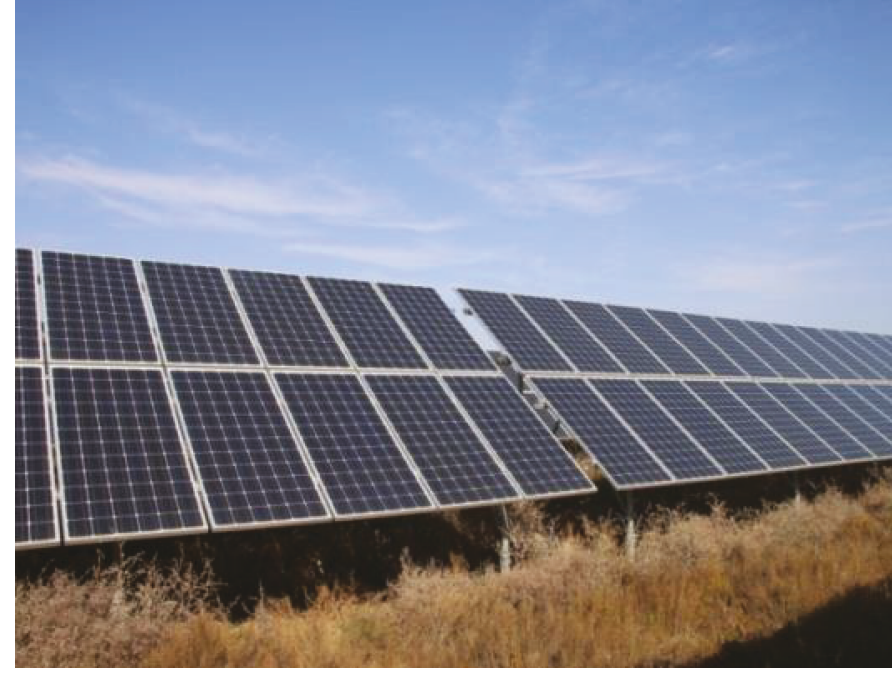

(a)

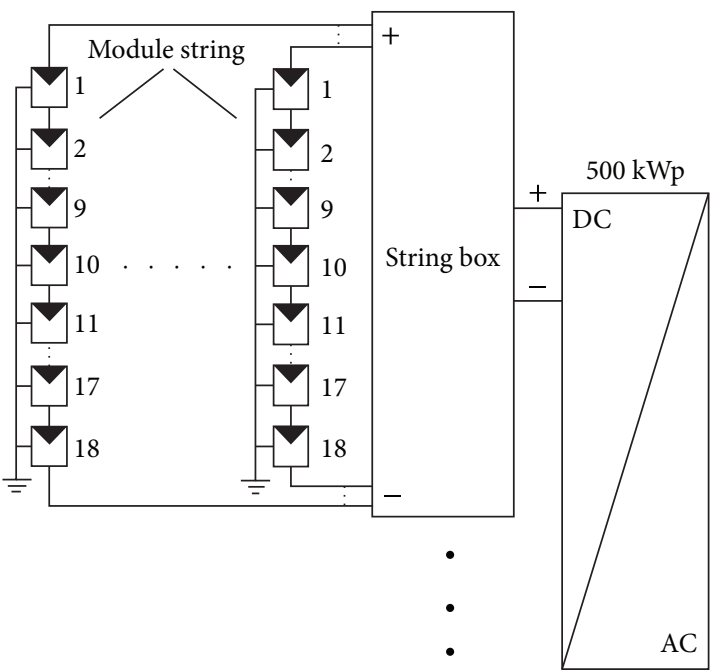

(b)

FIgURE 1: The scene of the $50 \mathrm{MWp}$ grid-connected photovoltaic power plant (a) and the circuit diagram of the subarray in the power plant (b).

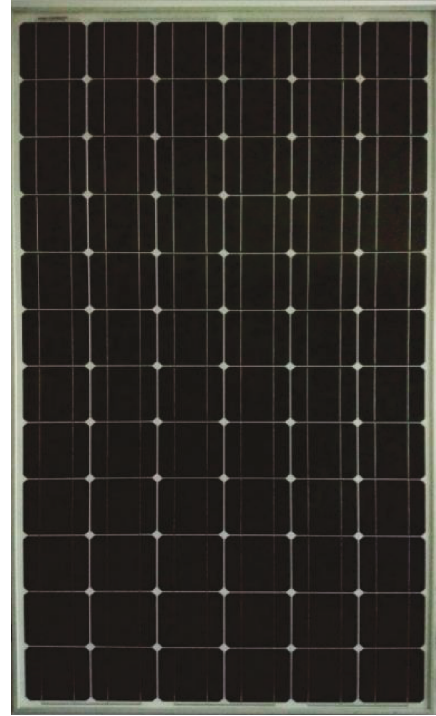

(a)

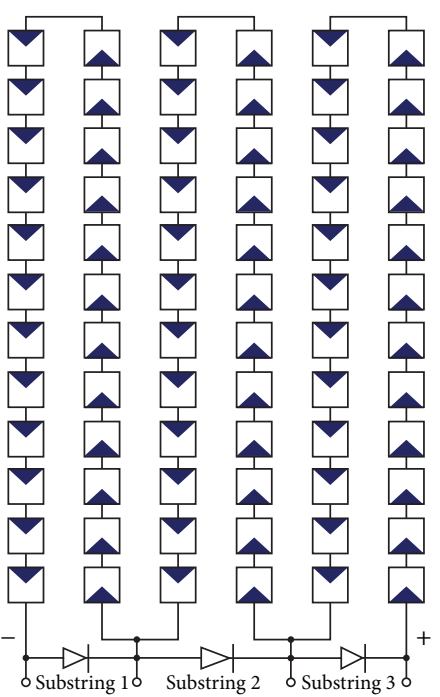

(b)

FIgURE 2: A photograph of the PID-affected solar module (a) and the circuit diagram of the solar module with 72 cells (b).

the thermographic and electroluminescence (EL) images for the PID-affected module string were revealed. The electrical performance of PID-affected modules also shows that the power degradation rate of these solar modules is related with the position of module installation in a module string. The new findings obtained in this paper can help identify and uncover the mystery of PID.

\section{Experiments and Methods}

In this work, the PID-affected solar modules come from the $50 \mathrm{MWp}$ crystalline silicon solar power plant located in China. Figure 1(a) shows the scene of the $50 \mathrm{MWp}$ grid-connected power plant, and Figure 1(b) shows the circuit diagram of the subarray in the power plant. Figure 2(a) shows a photograph of the PID-affected solar module, and Figure 2(b) shows the circuit diagram of the solar module with 72 solar cells. In the photovoltaic power plant, the eighteen solar modules are interconnected serially into a module string which is grounded by the aluminum frame of every module. Every solar module consists of 72 pieces of P-type monocrystalline silicon solar cells connected in a series, respectively. The cells feature an unpassivated full-area screen-printed aluminium back surface field with dimensions of $125 \times 125 \mathrm{~mm}^{2}$ and $70 \mathrm{~nm} \mathrm{SiNx}: \mathrm{H}$ antireflective coating [12]. 


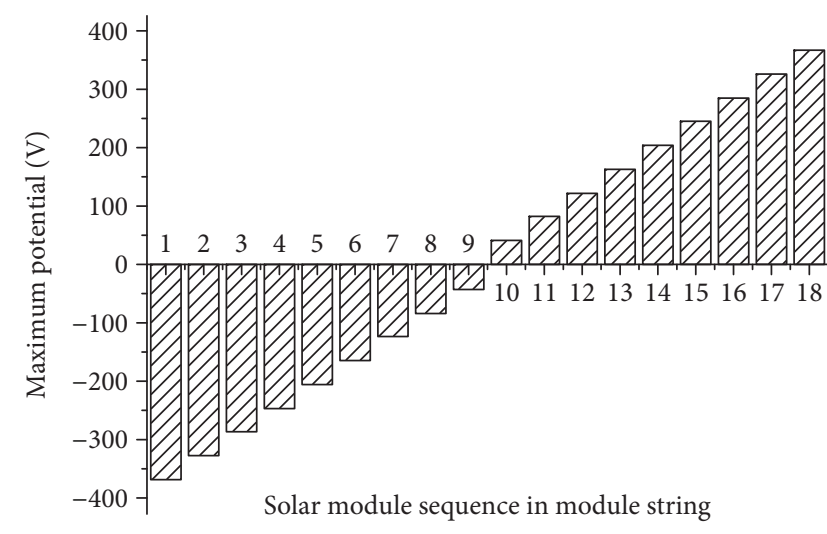

(a) Soon after grid-connection

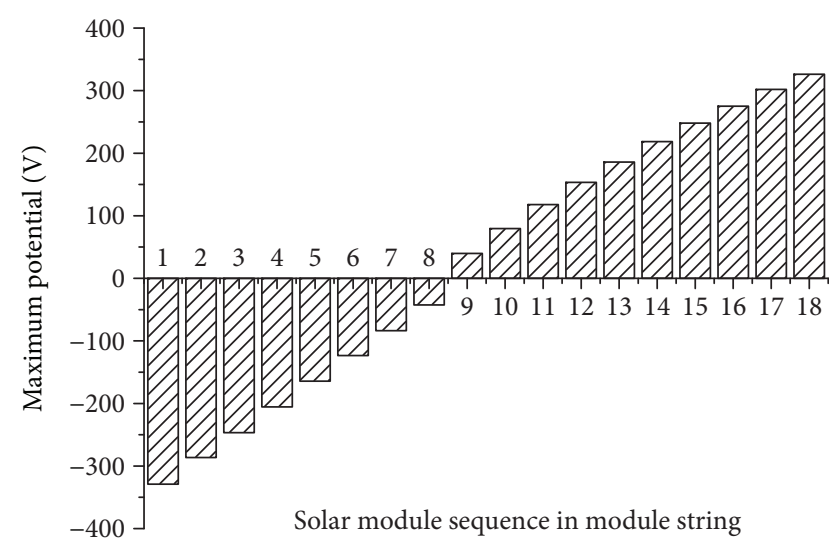

(b) After one year of operation

FIGURE 3: The measured potential difference of 18 modules from the same module string between the ground and the pole of module under an open circuit.

In order to get stable results, we chose an $\sim 25^{\circ} \mathrm{C}$ ambient temperature, more than $800 \mathrm{~W} / \mathrm{m}^{2}$ solar irradiation, and $\sim 60 \%$ relative humidity as test conditions in the field. Thermographic images of the solar modules were obtained by a T420 infrared camera with a resolution of $320 \times 240$ pixels and a spectral range of 7.5-13 $\mu \mathrm{m}$ (FLIR Systems Inc., T420). The sunlight exposure dose was measured by a YFJ-10 solar irradiation meter. The operation temperature of the solar module was measured by a thermocouple. The solar modules, which are from the PID-affected module string, were dismounted and marked in sequence. The performance of the solar modules was measured by a solar simulator (PASAN Sunsim 3C) under standard test conditions (STC: $1 \mathrm{~kW} / \mathrm{m}^{2}$ irradiance, $25^{\circ} \mathrm{C}$ module temperature and AM1.5 global spectrum). The solar simulator was calibrated by the standard module. The electroluminescence (EL) images of the solar modules were measured by an EL measurement system in the laboratory.

\section{Results and Discussion}

3.1. The Potential and the Neutral Point Shift of Module String. Figure 3 shows the maximum potential differences of the 18 modules from the same module string between the ground and cells of the solar module, which were measured in the field under an open circuit. The potential difference between the ground and cells depends on the number of solar modules, solar irradiation, and module temperature. Figure 3(a) shows the measured results of the maximum potential differences of the 18 modules from the same module string soon after the grid for the power plant was finished. Figure 3(b) shows the measured results of the maximum potential differences of the 18 modules from the same module string after one year of operation. From Figure 3, we find that the potential difference between the ground and cells is negative from module 1 to module 9 , and that the potential difference between the ground and cells is positive from module 10 to module 18 soon after the power plant was connected with the grid. After one year of operation, the neutral point of voltage in the module string moved forward to the positive pole. The potential difference between the ground and cells in module 9 evolved from the negative to positive. From module 9 to module 18, the closer the module gets to the negative pole of system, the more serious the degradation of open circuit voltage for module is [11]. Because of a transformerless inverter topology, the neutral point of the voltage in the module string moved forward to the positive pole when the aluminium frame of the solar modules is grounded. When the direction of the electric field of module 9 reverses after one year of operation, even if low positive voltage is applied to the PID-prone module, it could cause PID.

Comparing Figure 3(a) with Figure 3(b), the open circuit voltage of the module string becomes smaller for the same module string under nearly the same test conditions mentioned above. This is caused by PID of the module string. The negative potential versus the ground leads to an occurrence of PID.

The potential difference between the ground and cells creates an electric field on the surface of solar cells. Figure 4(a) shows the electric fields of 18 modules from the same module string soon after grid connection, and Figure 4(b) shows the electric fields of 18 modules after one year of operation.

From Figure 4, we find that only the electric field produced by the positive potential difference between the ground and cells could drive the metal ions, such as sodium ions driven into the PN junction which has existing stacking faults. This leads to damage of the PN junction and reduction of the open circuit voltage. This conclusion agrees with [13].

Figure 5 shows the direction of the electric field between the ground and solar cells of modules from the same module string. Soon after the plant was grid-connected, the direction of the electric field is directed from the solar cells to the $\mathrm{Al}$ frame for module 1 to module 9, as shown in Figure 5(a), while the direction of the electric field is reversed for module 10 to module 18, as shown in Figure 5(b). After one year of operation, the direction of the electric field for module 1 to module 8 is directed from the solar cells to the $\mathrm{Al}$ frame, while the direction of the electric field for module 9 to module 18 is reversed because of PID. This shows that the direction of the electric field of module 9 reverses after one year of 


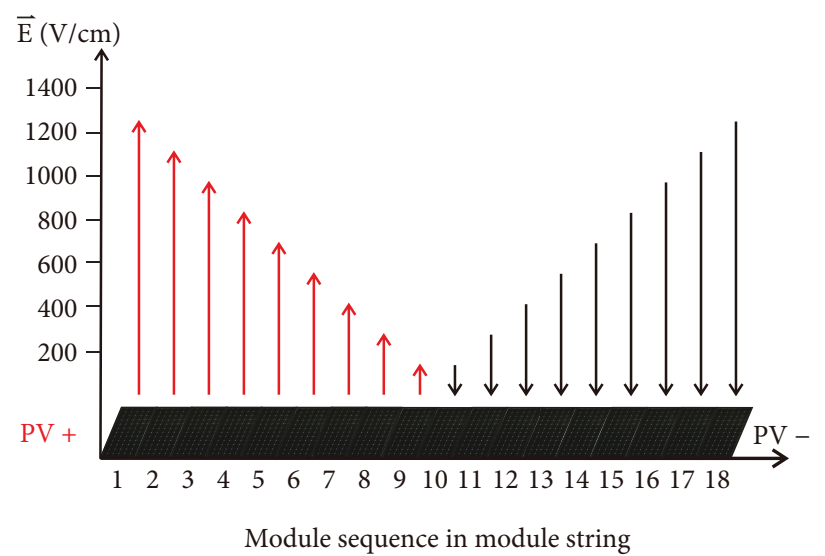

(a) Soon after grid-connection

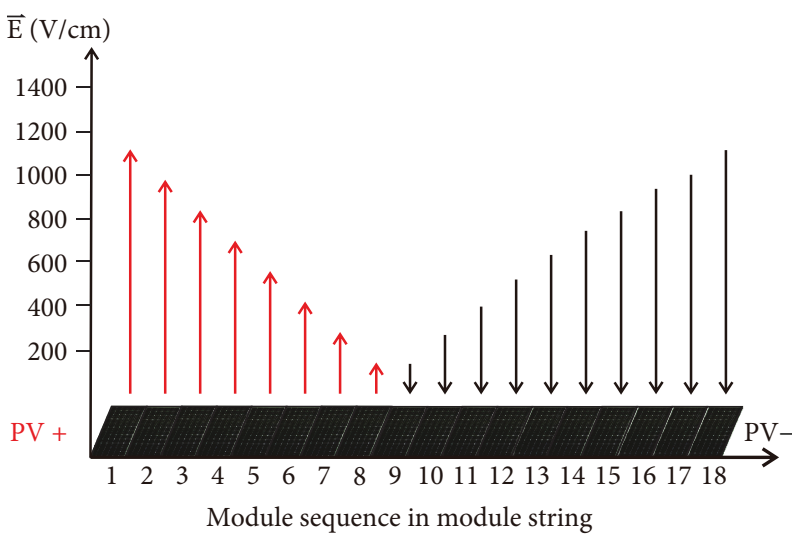

(b) After one year of operation

FIGURE 4: The electric fields of 18 modules from the same module string.

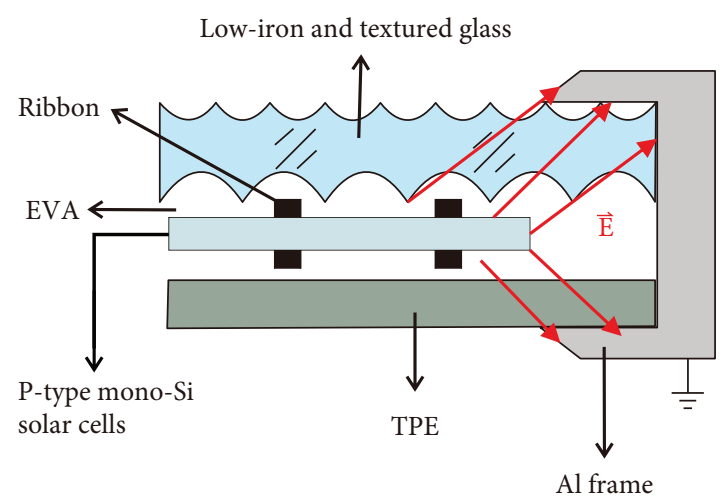

(a)

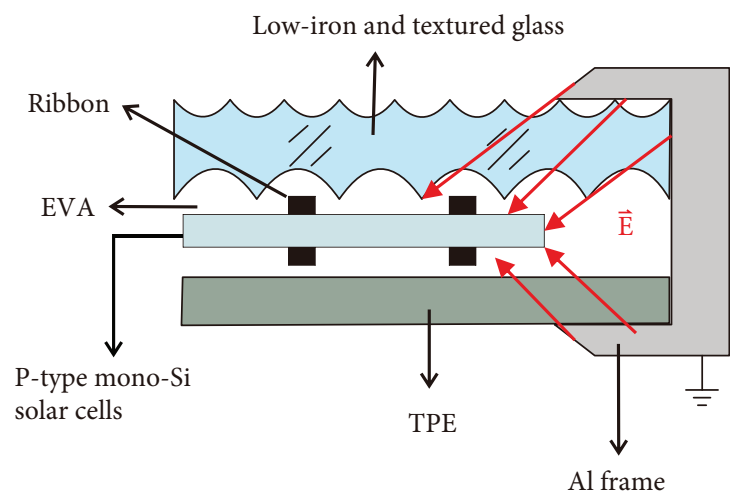

(b)

Figure 5: The direction of the electric field between the Al frame and solar cells of the module. (a) The direction of the electric field is directed from the solar cells to the Al frame. (b) The direction of the electric field is directed from the Al frame to the solar cells.

operation. This phenomenon means that the neutral point of voltage in a module string moved forward to the positive pole for a PID-affected module string as time goes on. If there does not exist an electric field produced by positive voltage applied to the cells, PID does not happen.

Based on the above analysis, we find that the positive potential difference between the ground and solar cells is a necessary condition for PID. To eliminate the electric field produced by the positive potential difference between the ground and cells is an effective measure to prevent PID.

\subsection{Thermographic Images of Solar Modules from the Same} PID-Affected Module String. The thermographic images of the solar modules from the same PID-affected module string are displayed in Figure 6. The photo was taken in the field under an ambient temperature of $\sim 20^{\circ} \mathrm{C}$, global irradiation of $\sim 800 \mathrm{~W} / \mathrm{m}^{2}$, and wind speed of $\sim 1 \mathrm{~m} / \mathrm{s}$. During image acquisition, the module strings were operated at maximum power point. The thermographic images of 18 solar modules had the highest temperature spot, where the junction box was built on the back. This is because the junction box affects the heat dissipation of the solar cells. From Figure 6, the thermographic images from module 1 to module 9 exhibit a

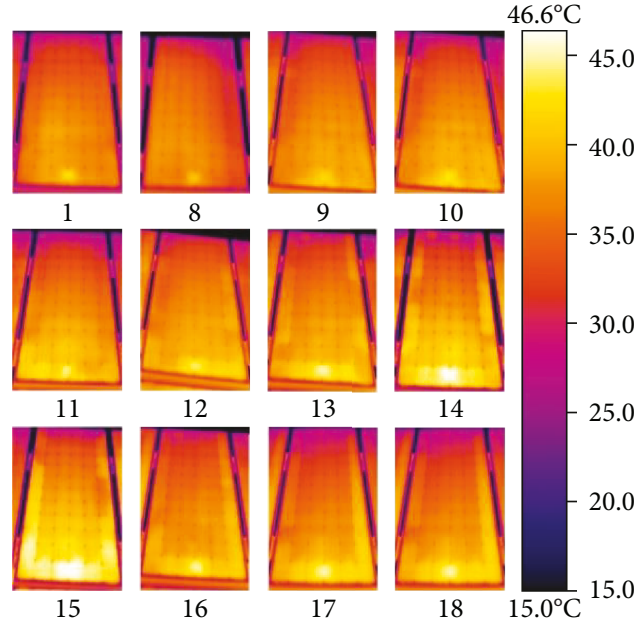

FIGURE 6: The thermographic images of solar modules from the same PID-affected module string.

homogeneous temperature distribution. No solar cells have an elevated temperature from module 1 to module 9. It is seemingly suggested that the modules from 1 to 9 are PID- 


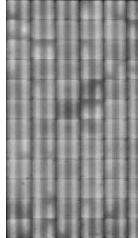

1

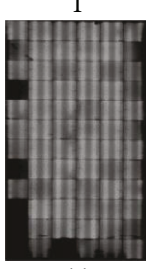

11

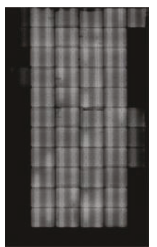

15

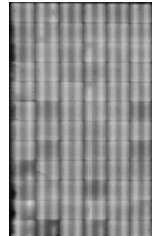

8

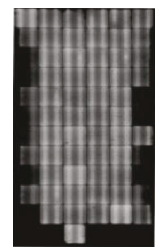

12

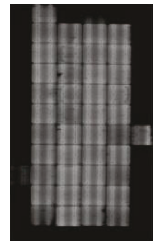

16

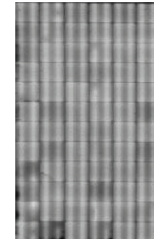

9

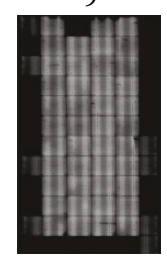

13

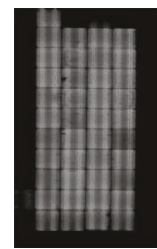

17

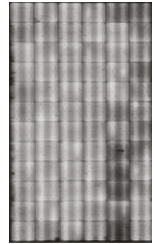

10

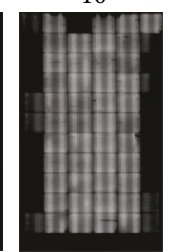

14

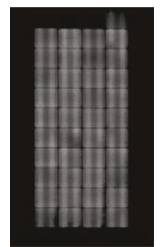

18
Figure 7: The electroluminescence images of solar modules dismounted from the same PID-affected module string.

free. This agrees with $[14,15]$. Instead, there are some solar cells with an elevated temperature from module 10 to module 18. The closer a module is to the negative pole, the greater is the number of cells with an elevated temperature. The thermographic images of solar modules from the same PIDaffected module string in the field show a regular degradation pattern. This is different with the results obtained in the laboratory $[16,17]$.

An interesting finding is that the temperature of the solar cells adjacent to the $\mathrm{Al}$ frame is higher than the middle cells in a PID-affected solar module. This is because the electric field adjacent to the $\mathrm{Al}$ frame is more intensive than the middle area; that is, the solar cells adjacent to the $\mathrm{Al}$ frame suffer more severe PID. The shunt resistance of PID-affected cells decreases dramatically; consequently, the dark current of the cells increases remarkably under illumination. The performance of the solar cells adjacent to the $\mathrm{Al}$ frame is inferior to the middle cells for a PID-affected solar module. So, a smaller portion of the sunlight is converted to electrical energy. Thus, PID-affected cells exhibit a higher temperature than sound ones.

3.3. Electroluminescence Images of Solar Modules Dismounted from the Same PID-Affected Module String. Figure 7 shows the electroluminescence images of solar modules dismounted from the same PID-affected module string in the power plant. The photos were taken indoors. From Figure 7, we find that the cells from modules 1 to 9 have the same EL intensity. From module 10 to module 18, some solar cells adjacent to the $\mathrm{Al}$ frame appear dark in the EL images, while the middle cells appear bright in a PID-affected solar module. The closer a module is to the negative pole, the greater is the number of dark cells in a PID-affected module. The darker the appearance of the solar cell is, the more serious is the power degradation caused by PID. The EL images of the solar modules dismounted from the same PID-affected module string yield a regular degradation pattern. Comparing Figure 6 with Figure 7, there is a good agreement between the electroluminescence images and the thermographic images, which exhibit a similar degradation pattern.

\subsection{The Power Degradation of Solar Modules Dismounted} from the Same PID-Affected Module String in the Field. Figure 8 shows the I-V and P-V curves of solar modules dismounted from the same PID-affected module string under standard test conditions. Table 1 shows the electrical parameters of module 8 before shipments and after one year of operation. The electrical parameters of module 9 before shipments and after one year of operation are presented in Table 2. From here, it can be observed that the power degradation rate is nearly the same from module 1 to module 8 . Module 9 has a $2.5 \%$ power drop after one year of operation, and it is higher than that of module 8 . This shows that the low positive potential difference between the ground and cells also leads to PID for a PID-prone solar module. This shows that the solar module slightly affected by PID could not be identified by the thermographic and electroluminescence images because of limitations in accuracy. The peak powers and open circuit voltages ( $\mathrm{Voc}$ ) of modules from 9 to 18 decrease remarkably in sequence. However, the short circuit currents of the PID-affected modules from the same module string have a slight change; this is consistent with some findings in the literature [18]. The closer a module is to the negative pole, the more serious is the power degradation of the PID-affected solar module.

Table 3 shows the electrical parameters for module 18 before shipments and after one year of operation. From Table 3, we found that the Voc of module 18 decreases from $45.01 \mathrm{~V}$ to $31.86 \mathrm{~V}$, and the peak power of module 18 decreases from $196.95 \mathrm{~W}$ to $92.06 \mathrm{~W}$, which was up to $53.26 \%$ power degradation after one year of operation. This shows that the potential-induced degradation leads to a catastrophic impact on the performance and energy yield of photovoltaic power plants.

\section{Conclusions}

This paper presents an investigation of PID-affected crystalline silicon solar modules from the field. For the first time, we find that low positive voltage could cause PID for PIDprone solar modules. For a PID-affected module string connected with the grid, the neutral point of voltage in a module string moved forward to the positive pole as time goes on. In a PID-affected photovoltaic power plant, the thermographic and electroluminescence images of the solar modules in a module string exhibit a regular degradation pattern simultaneously. This study also indicates that the power degradation rate of solar modules dismounted from the field is related with the position of the module installation in a module string. These new findings in this contribution can help identify and uncover the mystery of PID. The microanalysis of PID-affected cells dismounted from the field must be further 

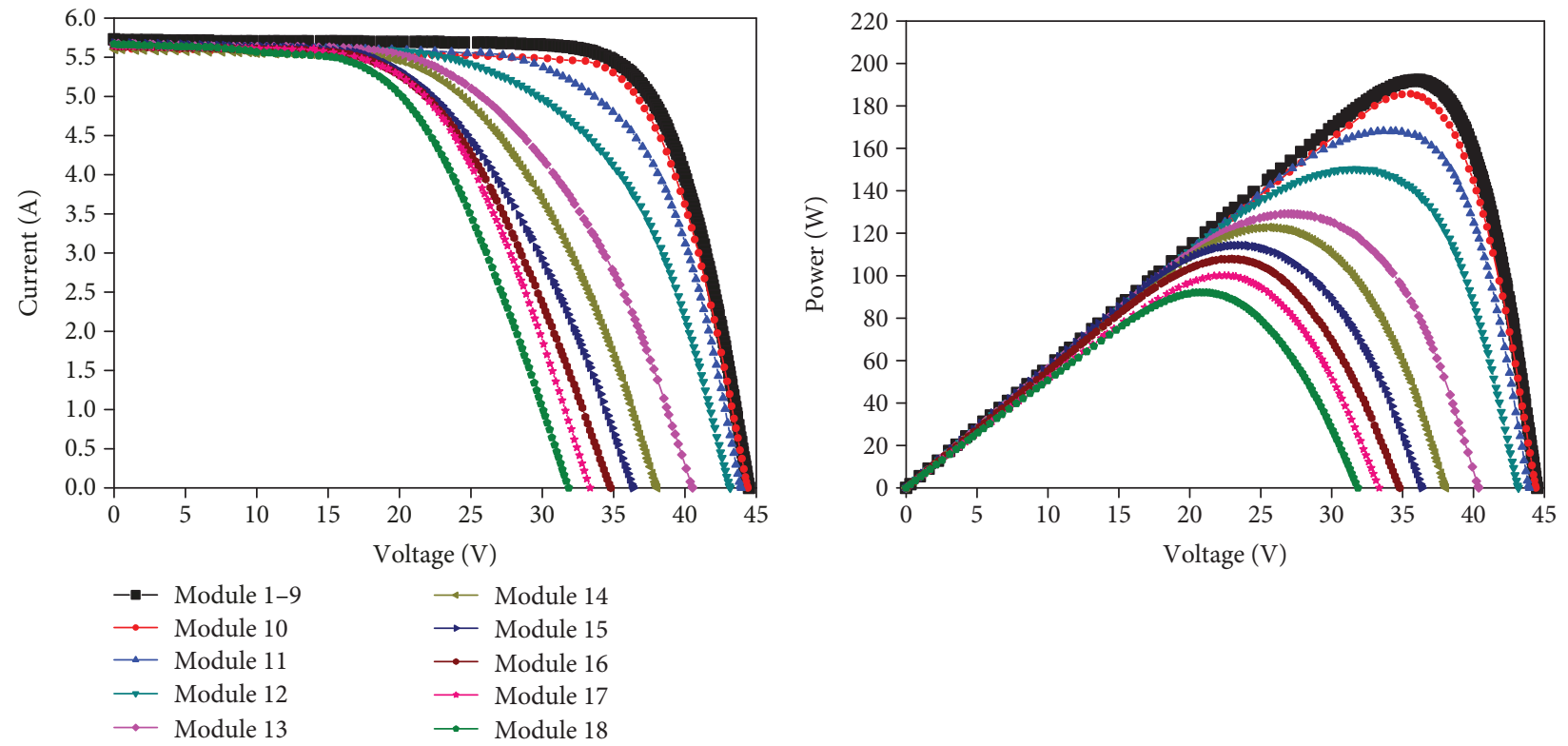

Figure 8: The I-V and P-V curves of solar modules disassembled from the same PID-affected module string in the power plant under standard test conditions.

TABLE 1: The electrical parameters for module 8 before shipments and after one year of operation.

\begin{tabular}{|c|c|c|c|c|c|c|}
\hline Module 8 & Voc $(\mathrm{V})$ & Isc (A) & $\mathrm{Vm}(\mathrm{V})$ & $\operatorname{Im}(\mathrm{A})$ & $\operatorname{Rsh}(\Omega)$ & $P_{\max }(\mathrm{W})$ \\
\hline Before shipments & 44.96 & 5.73 & 36.58 & 5.38 & 398.23 & 196.81 \\
\hline After one year of operation & 44.92 & 5.62 & 36.48 & 5.22 & 385.45 & 193.28 \\
\hline Degradation rate $(\%)$ & 0.09 & 1.92 & 0.27 & 2.97 & 3.21 & 1.79 \\
\hline
\end{tabular}

Test conditions: $1 \mathrm{~kW} / \mathrm{m}^{2}$ irradiance, $25^{\circ} \mathrm{C}$ module temperature and AM1.5 global spectrum.

TABLE 2: The electrical parameters of module 9 before shipments and after one year of operation.

\begin{tabular}{|c|c|c|c|c|c|c|}
\hline Module 9 & $\operatorname{Voc}(\mathrm{V})$ & Isc (A) & $\mathrm{Vm}(\mathrm{V})$ & $\operatorname{Im}(\mathrm{A})$ & $\operatorname{Rsh}(\Omega)$ & $P_{\max }(\mathrm{W})$ \\
\hline Before shipments & 45.02 & 5.78 & 36.52 & 5.40 & 389.27 & 197.19 \\
\hline After one year of operation & 44.87 & 5.65 & 36.32 & 5.29 & 360.54 & 192.27 \\
\hline Degradation rate $(\%)$ & 0.33 & 2.25 & 0.55 & 2.04 & 7.38 & 2.5 \\
\hline
\end{tabular}

Test conditions: $1 \mathrm{~kW} / \mathrm{m}^{2}$ irradiance, $25^{\circ} \mathrm{C}$ module temperature and AM1.5 global spectrum.

TABLE 3: The electrical parameters for module 18 before shipments and after one year of operation.

\begin{tabular}{lcccccc}
\hline Module 18 & Voc $(\mathrm{V})$ & Isc $(\mathrm{A})$ & $\mathrm{Vm}(\mathrm{V})$ & $\mathrm{Im}(\mathrm{A})$ & $\mathrm{Rsh}(\Omega)$ & $P_{\max }(\mathrm{W})$ \\
\hline Before shipments & 45.01 & 5.75 & 36.54 & 5.39 & 409.13 & 196.95 \\
After one year of operation & 31.86 & 5.60 & 19.30 & 4.77 & 182.36 & 92.06 \\
Degradation rate (\%) & 29.22 & 2.61 & 47.18 & 11.50 & 55.43 & 53.26 \\
\hline
\end{tabular}

Test conditions: $1 \mathrm{~kW} / \mathrm{m}^{2}$ irradiance, $25^{\circ} \mathrm{C}$ module temperature and AM1.5 global spectrum.

developed. An extension of this study is currently underway at our laboratory.

\section{Data Availability}

The data used to support the findings of this study are available from the corresponding author upon request.

\section{Conflicts of Interest}

The authors declare that they have no conflicts of interest.

\section{Acknowledgments}

The authors would like to thank the support of the National Key Technology R\&D Program (Grant no. 2015BAA09B01). 
This study was also supported by the Natural Science Foundation of China (Grant nos. 61376067 and 61274050) and the 2016 Grid State Science and Technology Program (NY71-16-027).

\section{References}

[1] D. C. Jordan, S. R. Kurtz, K. Vansant, and J. Newmiller, "Compendium of photovoltaic degradation rates," Progress in Photovoltaics: Research and Applications, vol. 24, no. 7, pp. 978-989, 2016.

[2] A. Ndiaye, A. Charki, A. Kobi, C. M. F. Kébé, P. A. Ndiaye, and V. Sambou, "Degradations of silicon photovoltaic modules: a literature review," Solar Energy, vol. 96, pp. 140-151, 2013.

[3] K. Mishina, A. Ogishi, K. Ueno et al., "Plasma-enhanced chemical-vapor deposition of silicon nitride film for high resistance to potential-induced degradation," Japanese Journal of Applied Physics, vol. 54, no. 8S1, article 08KD12, 2015.

[4] S. Jonai, K. Hara, Y. Tsutsui, H. Nakahama, and A. Masuda, "Relationship between cross-linking conditions of ethylene vinyl acetate and potential induced degradation for crystalline silicon photovoltaic modules," Japanese Journal of Applied Physics, vol. 54, no. 8S1, article 08KG01, 2015.

[5] S. Pingel, O. Frank, M. Winkler et al., "Potential induced degradation of solar cells and panels," in 201035 th IEEE Photovoltaic Specialists Conference, pp. 2817-2822, Honolulu, HI, USA, June 2010.

[6] M. Koehl and S. Hoffmann, "Impact of rain and soiling on potential induced degradation," Progress in Photovoltaics: Research and Applications, vol. 24, no. 10, pp. 1304-1309, 2016.

[7] B. Ziebarth, M. Mrovec, C. Elsasser, and P. Gumbsch, "Potential-induced degradation in solar cells: electronic structure and diffusion mechanism of sodium in stacking faults of silicon," Journal of Applied Physics, vol. 116, no. 9, article 093510, 2014.

[8] V. Naumann, D. Lausch, A. Hähnel et al., "Explanation of potential-induced degradation of the shunting type by $\mathrm{Na}$ decoration of stacking faults in Si solar cells," Solar Energy Materials and Solar Cells, vol. 120, pp. 383-389, 2014.

[9] S. P. Harvey, J. Moseley, A. Norman et al., "Investigating PID shunting in polycrystalline silicon modules via multiscale, multitechnique characterization," Progress in Photovoltaics: Research and Applications, vol. 26, no. 6, pp. 377-384, 2018.

[10] H. Wang, P. Zhao, H. Yang, J. Chang, D. Song, and S. Sang, "Performance variation of dark current density-voltage characteristics for PID-affected monocrystalline silicon solar modules from the field," Microelectronics Reliability, vol. 81, pp. 320-327, 2018.

[11] H. Yang, F. Wang, H. Wang, J. Chang, D. Song, and C. Su, "Performance deterioration of P-type single crystalline silicon solar modules affected by potential induced degradation in photovoltaic power plant," Microelectronics Reliability, vol. 72, pp. 18-23, 2017.

[12] H. Yang, H. Wang, D. Cao, D. Sun, and X. Ju, "Analysis of power loss for crystalline silicon solar module during the course of encapsulation," International Journal of Photoenergy, vol. 2015, Article ID 251615, 5 pages, 2015.

[13] J. Oh, S. Bowden, and G. S. Tamizhmani, "Potential-induced degradation (PID): incomplete recovery of shunt resistance and quantum efficiency losses," IEEE Journal of Photovoltaics, vol. 5, no. 6, pp. 1540-1548, 2015.
[14] C. Buerhop-Lutz, R. Weibmann, H. Scheuerpflug, R. Auer, and C. J. Brabec, "Quality control of PV-modules in the field using a remote-controlled drone with an infrared camera," in Proceedings of the 27th European Photovoltaic Solar Energy Conference and Exhibition (EU PVSEC 2012) 2012, pp. 3370 3373, Frankfurt, Germany, 2012.

[15] T. Kaden, K. Lammers, and H. J. Moller, "Power loss prognosis from thermographic images of PID affected silicon solar modules," Solar Energy Materials and Solar Cells, vol. 142, pp. 2428, 2015.

[16] M. B. Koentopp, M. Krober, and C. Taubitz, "Toward a PID test standard: understanding and modeling of laboratory tests and field progression," IEEE Journal of Photovoltaics, vol. 6, no. 1, pp. 252-257, 2016.

[17] P. Hacke, S. Spataru, K. Terwilliger et al., "Accelerated testing and modeling of potential-induced degradation as a function of temperature and relative humidity," IEEE Journal of Photovoltaics, vol. 5, no. 6, pp. 1549-1553, 2015.

[18] F. Martínez-Moreno, G. Figueiredo, and E. Lorenzo, "In-thefield PID related experiences," Solar Energy Materials and Solar Cells, vol. 174, pp. 485-493, 2018. 

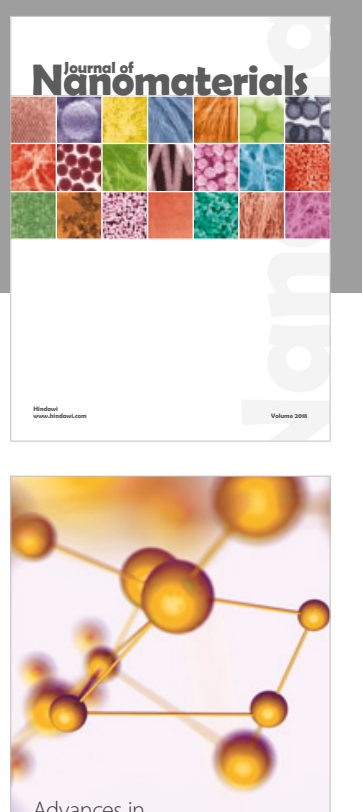

Physical Chemistry
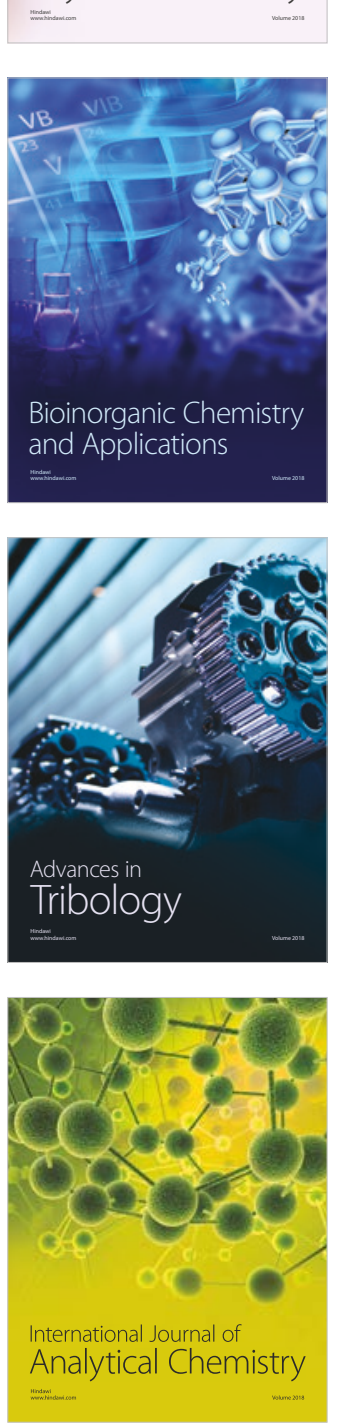

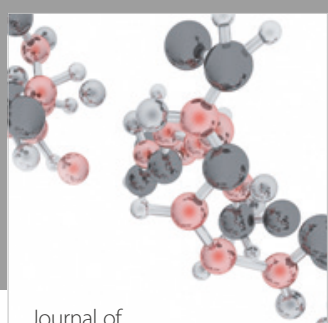

Analytical Methods

in Chemistry

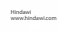

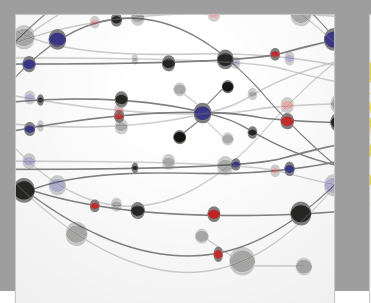

The Scientific World Journal

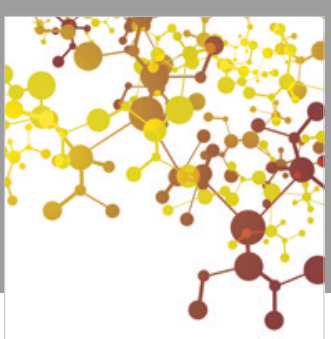

Journal of

Applied Chemistry
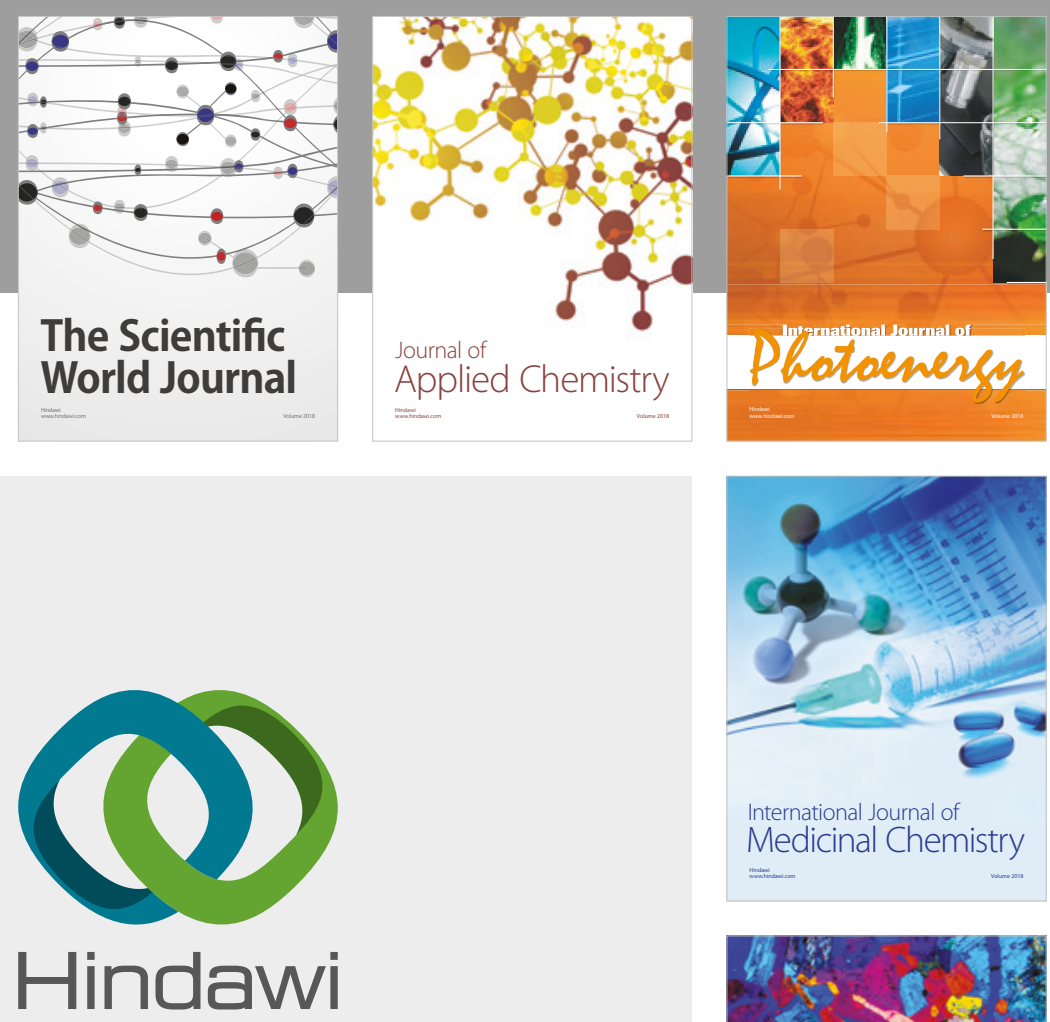

Submit your manuscripts at

www.hindawi.com
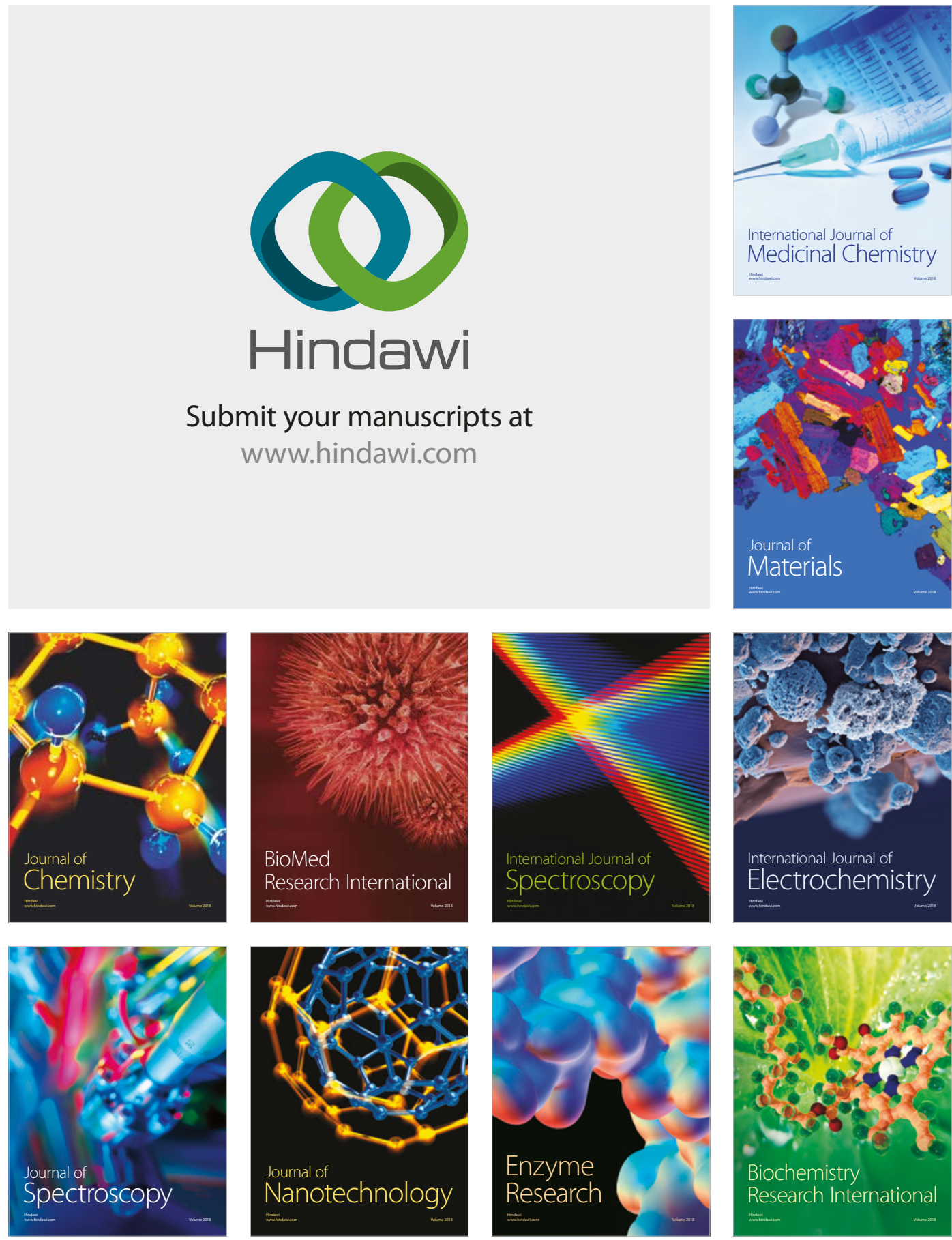
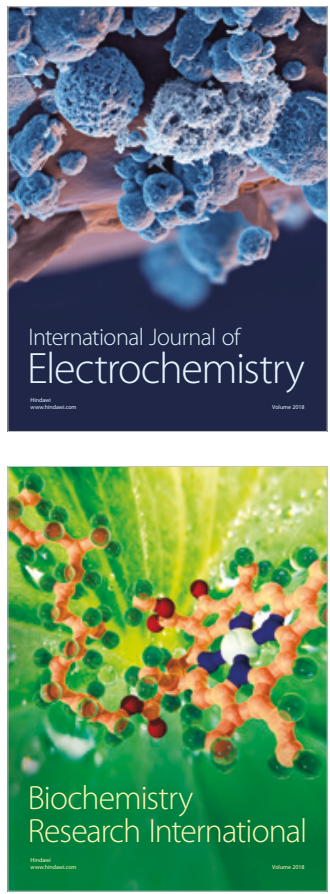